\section{Factores sociales del estado de salud autorreportado de personas mayores, en Chile}

\author{
SANDRA ALVEAR ${ }^{1, \mathrm{a}}$, PATRICIA RODRÍGUEZ ${ }^{1, \mathrm{~b}}$, \\ CAROLINA RIVEROS ${ }^{2, \mathrm{c}}$, ÁNGELA ARENAS ${ }^{2, \mathrm{~d}}$, JORGE CANTEROS ${ }^{3}$
}

\section{Factors influencing self-reported health in Chilean older people}

Background: Self-reported health is subjective and depends on external factors such as socioeconomic status, presence of chronic diseases and working status, among others. Aim: To determine which factors influence self-reported health among older people in Chile. Material and Methods: A secondary analysis of the National Socioeconomic Characterization survey done in 2015. A dichotomous response model was used classifying health status as good or bad. A logit regression model was carried out. Results: The model had a good calibration and correctly classified 72 and $68 \%$ of men and women, respectively. The main factors that influenced health status self-perception were: not having health problems; having undergone a mental health interview, to receive supplemental nutrition, education, to have a productive work; and to having a social network. Conclusions: There are health, cultural, economic and environmental factors that influence self-perceived health status.

(Rev Med Chile 2019; 147: 1407-1414)

Key words: Frail Elderly; Healthy Aging; Logistic Models; Public Health; Self Report.

\author{
${ }^{1}$ Facultad de Economía y \\ Negocios. Universidad de Talca. \\ Talca, Chile. \\ ${ }^{2}$ Facultad de Ciencias Jurídicas y \\ Sociales. Universidad de Talca. \\ Talca, Chile. \\ ${ }^{3}$ Unidad de Cuidados Intensivos. \\ Hospital de Curicó. Curicó, Chile. \\ ${ }^{a}$ Contador Público y Auditor. PhD \\ en Ciencias de la Educación. \\ ${ }^{b}$ Contador Público y Auditor. \\ PhD en Economía y Dirección de \\ Empresas. \\ 'Abogada. PhD en Derecho.

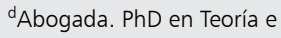 \\ Historia del Derecho Europeo. \\ Investigación, financiada por \\ CONICYT mediante el proyecto \\ FONDEF ID16AM0006: El \\ maltrato estructural a las \\ personas mayores en Chile. \\ Formulación de un índice \\ multidimensional (2017-2019). \\ Los autores declaran no tener \\ conflictos de interés. \\ Recibido el 30 de agosto \\ de 2018, aceptado el 13 de \\ noviembre de 2019. \\ Correspondencia a: \\ Sandra Alvear \\ Universidad de Talca. \\ Casilla 721. Talca, Chile. \\ salvear@utalca.cl
}

\section{I}

a sociedad chilena vivencia un envejecimiento poblacional que requiere ser enfrentado con un enfoque sistémico y holístico, sin embargo, la evidencia muestra que a nivel país aún se presentan diversos desafíos ${ }^{1}$.

Para planificar políticas públicas asociadas al envejecimiento de la población, desde la evaluación en salud, se pueden plantear dos miradas complementarias. Desde el punto de vista clínico, incorporando aspectos objetivos en salud. Desde la perspectiva de calidad de vida relativa a la salud, basadas en la percepción subjetiva relacionada con el bienestar y la percepción ${ }^{2}$, de ahí la importancia de contar con este tipo de estudio en los adultos mayores, en adelante mayores.

El estado de salud autorreportado se basa en criterios subjetivos, que incluye aspectos bio- lógicos, psicológicos y sociales, influenciados por la condición socioeconómica, género, edad, presencia de enfermedades crónicas, ocupación o no ocupación, entre otros factores ${ }^{3-5}$. El estado de salud autorreportado es un indicador de salud global y permite predecir necesidades asistenciales, la organización de programas, que reflejan elementos de salud y también sociales ${ }^{6}$.

Por consiguiente, es relevante contar con modelos de decisión que evalúen las políticas públicas implementadas, con un enfoque multidimensional ${ }^{7}$, específicamente para los mayores, incorporando una mirada subjetiva, tal como el estado de salud autorreportado. La Encuesta de Caracterización Socioeconómica Nacional (Casen) $2015^{8}$ incluyó una pregunta que mide el estado de salud autorreportado de los indivi- 
duos. Del total de mayores en estudio, alrededor de $82,5 \%$ de ellos califica su estado de salud con nota igual o superior a 4 , es decir, de regular a bueno. La autovaloración positiva del estado de salud expresa la sensación de bienestar individual ${ }^{9}$ y la mala percepción es un buen predictor de mortalidad $^{10}$.

El presente estudio tiene como objetivo evaluar en el estado de salud autorreportado por los mayores en Chile y analizar los factores asociados a ella.

\section{Material y Métodos}

Se trata de un análisis de datos secundarios, obtenidos de la encuesta Casen 2015, los que son de uso público y de propiedad del Estado de Chile 8 .

Esta encuesta es ampliamente usada para investigación científica en el país, sin requerimiento de evaluación de Comité de Ética. Los datos de los mayores se encuentran anonimizados en la encuesta, por ello no fue necesario solicitar consentimiento informado ${ }^{11}$.

Como criterio de inclusión se consideró a los mayores, cuya edad es igual o superior a 60 años, según la Ley No 19.828 del año 2015, que fija la adultez mayor en 60 años o más ${ }^{12}$. Dado que la Casen 2015 es una encuesta a hogares con diseño muestral probabilístico, se empleó un expansor regional (expr), un factor que expande a la proyección de población regional ${ }^{8}, y$, así, se tiene validez sobre la población objetivo, la totalidad del país, y corrige los errores estándares. Se conformó una muestra de 1.743 .112 mayores, $59 \%$ de sexo femenino y $41 \%$ masculino.

La variable dependiente del estudio es dicotómica y representa la autovaloración del estado de salud de los mayores, obtenida a partir de la respuesta dada a la siguiente pregunta "Ahora, en una escala de 1 a 7 , donde 1 corresponde a muy mal y 7 a muy bien, ¿qué nota le pondría a su estado de salud actual?". El análisis de respuestas la agrupamos en dos categorías, según la escala de evaluación culturalmente aceptada en Chile: Autovaloración positiva $=1 \mathrm{y}$ autovaloración negativa $=0$. Se consideró como autovaloración positiva las respuestas 4, 5, 6 y 7 y como autovaloración negativa las respuestas 1,2 y 3 . Se realizó un segundo análisis agrupando las respuestas 6 y 7 para autovaloración positiva. Los resultados no cambian y se mantiene la robustez del modelo.
Como variables independientes definimos de esta forma: Sexo, otorgando valor 1 para hombre y 0 para mujer. Nacionalidad, con valor 1 para chilena y 0 para extranjera. Educación, fue categorizada en básica, media, superior y sin escolaridad, tomando el valor 1 si se reportaba escolaridad y 0 si no lo reportaba. Trabajo, tomando el valor 1 si hubiera trabajado la semana anterior a la encuesta y 0 si no lo hubiera hecho. Sistema previsional, con valor 1 si la persona pertenece al sistema previsional Fonasa o era indigente y 0 si la persona cotiza en Isapre u otro sistema. Patología, asignamos el valor 1 , si la persona en los últimos meses ha estado en tratamiento médico por patología cardiovascular, trastorno del ánimo (patologías descritas en forma independiente en la encuesta), otra condición de salud y toma el valor 0 , si no ha estado en tratamiento médico. Redes de apoyo, considerando el valor 1 , si la persona, debido a su estado de salud, alguna persona del hogar le prestó ayuda y asignando el valor 0 si no recibió ayuda. Condición permanente, que fue expresado en la encuesta de la manera que se utiliza en el trabajo, asignamos el valor 1 , si la persona tenía dificultad física o de movilidad, mudez o alteración del habla, dificultad psiquiátrica, dificultad mental o intelectual, sordera o dificultad para oír, ceguera o dificultad para ver y toma el valor 0 , si no se reportaba dicha condición. Problemas de salud, con valor 1 , si la persona en los últimos tres meses presentó un problema de salud, enfermedad o accidente, y 0 si no presentó problemas de salud. Nutrición, asignamos el valor 1, si la persona, en los últimos tres meses, había recibido o retirado, gratuitamente alimentos del consultorio, y 0 si no hubiera retirado dichos alimentos. Consulta mental, asignando el valor 1 , si la persona, en los últimos tres meses realizó consulta mental, y 0 si no realizó consulta mental. Exámenes del laboratorio, toma el valor 1 , si la persona, en los últimos tres meses previos a la encuesta hubiera realizado exámenes de laboratorio y 0 si no si no se hubiera realizado exámenes. Quintil de ingresos, variable dividida en cinco subvariables, quinta parte o $20 \%$ de los hogares nacionales ordenados en forma ascendente de acuerdo al ingreso autónomo per cápita del hogar, donde el primer quintil representa el $20 \%$ más pobre del país y el quinto quintil el $20 \%$ más rico de estos hogares, cada una de ellas toma el valor 1, si la persona pertenece al tramo de ingresos que se analiza y 0 si 
no pertenece. El país está dividido en 15 regiones geográficas y analizamos la relevancia de vivir en una determinada región, tomando el valor 1 si la persona habita en la región y 0 si no habita en ella.

El análisis estadístico de los datos se realizó con el apoyo del software Stata13. El regresor se refiere a un modelo logit y su especificación es:

$$
y_{i}=F\left(\begin{array}{c}
\beta_{0}+\beta_{1} \text { Nacionalidad }_{i}+\beta_{2} \text { Educación }_{i}+ \\
\beta_{3} \text { Trabajo }_{i}+\beta_{4} \text { Sistema Previsional }_{i}+ \\
\beta_{5} \text { Redes deapoyo }_{i}+\beta_{6} \text { Problemas de Salud }_{i} \\
+\beta_{7} \text { Alimentación }_{i}+\beta_{6} \text { Consulta Mental }_{i}+ \\
\beta_{6} \text { Exámenes delaboratorio }_{i}+\sum_{j=10}^{15} \beta_{j} \text { Patología }_{j i} \\
+\sum_{k=16}^{21} \beta_{k} \text { Condición permanente }_{k i}+ \\
\sum_{l=22}^{27} \beta_{l} \text { QuintildeIngreso }_{l i}+\sum_{m=28}^{43} \beta_{m} \text { Región }_{m i}
\end{array}\right)+\varepsilon_{i}
$$

Donde $F($.) corresponde la función de distribución acumulada que se asume logística ${ }^{13}$, la cual cumple el rol de enlace entre los determinantes y la probabilidad de tener buena salud autorreportada por los mayores. Por lo tanto, el modelo logit a estimar corresponde a:

$$
y_{i}=\frac{e^{\sum_{p=0}^{34} \beta_{p} X_{p i}}}{1+e^{\sum_{p=0}^{34} \beta_{p} X_{p i}}}+\varepsilon_{i}
$$

Donde $X_{p i}$ corresponde a las 43 determinantes previamente especificadas.

La diferencia entre los valores predichos y observados se resumen en el estadístico chi cuadrado de Pearson $\chi^{2}$, tomando el valor $(97170,44)$ para los hombres y $(130914,35)$ para las mujeres, ambos con 34 grados de libertad. El estadístico tiene un valor-p menor a 0,005 , en tanto, el modelo presenta un buen ajuste a los datos. En general, el modelo clasifica correctamente $64,97 \%$ y $64,75 \%$ de los casos, para hombres y mujeres, respectivamente (Tabla 1).

\section{Resultados}

En general, no existen diferencias significativas entre el estado de salud autorreportado por mujeres y hombres. Las mujeres reportaron un buen estado de salud en $48 \%$ de las encuestadas y los hombres lo hicieron en 50\% (Tabla 2).

Los mayores que reportaron preferentemente un buen estado de salud: trabajaban o habían trabajado en la última semana (61\%), su sistema previsional era privado (59\%), poseían dificultad física $(55 \%)$, presentaban problemas de salud (56\%), no se habían realizado exámenes de laboratorio $(53 \%)$ y estaban clasificados en los quintiles de ingresos 4 y $5(52 \%, 61 \%)$ (Tabla 2).

Los mayores que reportan preferentemente un mal estado de salud: eran de nacionalidad extranjera (56\%), no habían asistido al sistema

Tabla 1. Evaluación del modelo de regresión logística

\begin{tabular}{|lcc|}
\hline & Hombre & Mujer \\
\hline Log likelihood & $-449223,61$ & $-644197,8$ \\
\hline Número de observaciones & 718.305 & 1.025 .007 \\
\hline LR $\chi^{2}$ (34) & 97170,44 & 130914,35 \\
\hline Probabilidad $>\chi^{2}$ & 0,0000 & 0,0000 \\
\hline LR (34) & 97170,44 & 130914,35 \\
\hline Probabilidad > LR & 0,0000 & 0,0000 \\
\hline McFadden's R2 & 0,098 & 0,092 \\
\hline Count R2 & 0,650 & 0,647 \\
\hline Akaike (AIC) & 1,251 & 1,257 \\
\hline Adj Conut R2 & 0,295 & 0,269 \\
\hline R2 Cox \& Snell & 0,1265 & 0,1199 \\
\hline R2 adj. Nagelkerke & 0,1687 & 0,1599 \\
\hline Sensibilidad & $71,52 \%$ & $68,28 \%$ \\
\hline Especificidad & $58,34 \%$ & $61,45 \%$ \\
\hline Correctamente clasificados & $64,97 \%$ & $64,75 \%$ \\
\hline
\end{tabular}

Fuente: Elaboración propia a partir de los datos de la Casen, 2015. LR: Likelihood-ratio test after estimation. Prob: Probabilities. McFadden's 2: choice model, which is a specific case of the more general conditional logistic regression model fit by clogit. Count: Count R-Squared does not approach goodness of fit in a way comparable to any OLS approach. It transforms the continuous predicted probabilities into a binary variable on the same scale as the outcome variable (0-1) and then assesses the predictions as correct or incorrect. Akaike: Estimates stats reports model-selection statistics, including the Akaike information criterion (AIC) and the Bayesian information criterion (BIC). These measures are appropriate for maximum likelihood models. Adj Conut: The Adjusted Count R-Square mirrors approach 2 from the list above. This adjustment is unrelated to the number of predictors and is not comparable to the adjustment to OLS or McFadden's R-Squareds. R2 Cox\&Snell: The ratio of the likelihoods reflects the improvement of the full model over the intercept model (the smaller the ratio, the greater the improvement). R2 adj. Nagelkerke: It adjusts Cox \& Snell's so that the range of possible values extends to 1.To achieve this, the Cox \& Snell R-squared is divided by its maximum possible value, $1-\mathrm{L}($ MIntercept)2/N. 
Tabla 2. Descripción de las variables

\begin{tabular}{|c|c|c|c|c|c|c|}
\hline \multirow[b]{2}{*}{ Sexo } & \multirow[b]{2}{*}{$\begin{array}{l}\text { Mujer } \\
\text { Hombre }\end{array}$} & \multicolumn{2}{|c|}{$\begin{array}{l}\text { Buen estado de salud } \\
\text { autorreportada }\end{array}$} & \multicolumn{2}{|c|}{$\begin{array}{l}\text { Mal estado de salud } \\
\text { autorreportada }\end{array}$} & \multirow{2}{*}{$\begin{array}{r}\text { Total } \\
1.024 .750 \\
718.362\end{array}$} \\
\hline & & $\begin{array}{l}494.209 \\
361.121\end{array}$ & $\begin{array}{l}48 \% \\
50 \%\end{array}$ & $\begin{array}{l}530.541 \\
357.241\end{array}$ & $\begin{array}{l}52 \% \\
50 \%\end{array}$ & \\
\hline Nacionalidad & $\begin{array}{l}\text { Chileno(a) } \\
\text { Extranjero(a) }\end{array}$ & $\begin{array}{r}850.952 \\
4.684\end{array}$ & $\begin{array}{l}49 \% \\
44 \%\end{array}$ & $\begin{array}{r}881.773 \\
5.903\end{array}$ & $\begin{array}{l}51 \% \\
56 \%\end{array}$ & $\begin{array}{r}1.018 .536 \\
6.471\end{array}$ \\
\hline Educación & $\begin{array}{l}\text { Asistió a la educación formal } \\
\text { No asistió a la educación formal }\end{array}$ & $\begin{array}{r}809.369 \\
46.312\end{array}$ & $\begin{array}{l}50 \% \\
37 \%\end{array}$ & $\begin{array}{r}809.303 \\
78.328\end{array}$ & $\begin{array}{l}50 \% \\
63 \%\end{array}$ & $\begin{array}{r}1.618 .672 \\
124.640\end{array}$ \\
\hline Trabajo & $\begin{array}{l}\text { Con trabajo } \\
\text { Sin trabajo }\end{array}$ & $\begin{array}{l}242.472 \\
613.209\end{array}$ & $\begin{array}{l}61 \% \\
46 \%\end{array}$ & $\begin{array}{l}157.792 \\
730.439\end{array}$ & $\begin{array}{l}39 \% \\
54 \%\end{array}$ & $\begin{array}{r}399.664 \\
1.343 .648\end{array}$ \\
\hline $\begin{array}{l}\text { Sistema } \\
\text { previsional }\end{array}$ & $\begin{array}{l}\text { Fonasa } \\
\text { Privado }\end{array}$ & $\begin{array}{l}747.107 \\
108.574\end{array}$ & $\begin{array}{l}48 \% \\
59 \%\end{array}$ & $\begin{array}{r}811.207 \\
76.424\end{array}$ & $\begin{array}{l}52 \% \\
41 \%\end{array}$ & $\begin{array}{r}1.558 .314 \\
184.998\end{array}$ \\
\hline Patología & $\begin{array}{l}\text { Cardiovascular } \\
\text { Trastornos del ánimo } \\
\text { Otra patología } \\
\text { Sin patología }\end{array}$ & $\begin{array}{l}428.269 \\
482.354 \\
410.941 \\
350.412\end{array}$ & $\begin{array}{l}50 \% \\
48 \% \\
51 \% \\
44 \%\end{array}$ & $\begin{array}{l}421.659 \\
514.700 \\
397.473 \\
439.034\end{array}$ & $\begin{array}{l}50 \% \\
52 \% \\
49 \% \\
56 \%\end{array}$ & $\begin{array}{l}849.928 \\
997.054 \\
808.414 \\
780.446\end{array}$ \\
\hline $\begin{array}{l}\text { Redes de } \\
\text { apoyo }\end{array}$ & $\begin{array}{l}\text { Tiene ayuda } \\
\text { No tiene ayuda }\end{array}$ & $\begin{array}{l}755.537 \\
100.144\end{array}$ & $\begin{array}{l}56 \% \\
26 \%\end{array}$ & $\begin{array}{l}603.021 \\
284.610\end{array}$ & $\begin{array}{l}44 \% \\
74 \%\end{array}$ & $\begin{array}{r}1.358 .558 \\
384.754\end{array}$ \\
\hline $\begin{array}{l}\text { Condición } \\
\text { permanente }\end{array}$ & $\begin{array}{l}\text { Dificultad física } \\
\text { Sordera o dificultad para oír } \\
\text { Ceguera o dificultad para ver } \\
\text { Otra condición permanente } \\
\text { Sin condición permanente }\end{array}$ & $\begin{array}{l}757.390 \\
822.280 \\
826.634 \\
839.649 \\
176.771\end{array}$ & $\begin{array}{l}55 \% \\
49 \% \\
49 \% \\
50 \% \\
31 \%\end{array}$ & $\begin{array}{l}611.733 \\
848.788 \\
845.575 \\
854.039 \\
390.389\end{array}$ & $\begin{array}{l}45 \% \\
51 \% \\
51 \% \\
50 \% \\
69 \%\end{array}$ & $\begin{array}{r}1.369 .123 \\
1.671 .068 \\
1.672 .209 \\
1.693 .688 \\
567.160\end{array}$ \\
\hline $\begin{array}{l}\text { Problemas de } \\
\text { salud }\end{array}$ & $\begin{array}{l}\text { Sin problemas de salud } \\
\text { Con problemas de salud }\end{array}$ & $\begin{array}{l}290.934 \\
564.747\end{array}$ & $\begin{array}{l}40 \% \\
56 \%\end{array}$ & $\begin{array}{l}437.187 \\
450.444\end{array}$ & $\begin{array}{l}60 \% \\
44 \%\end{array}$ & $\begin{array}{r}728.121 \\
1.015 .191\end{array}$ \\
\hline Nutrición & $\begin{array}{l}\text { Recibe alimentos PACAM } \\
\text { No recibe alimentos PACAM }\end{array}$ & $\begin{array}{l}212.187 \\
643.494\end{array}$ & $\begin{array}{l}43 \% \\
51 \%\end{array}$ & $\begin{array}{l}276.620 \\
611.011\end{array}$ & $\begin{array}{l}57 \% \\
49 \%\end{array}$ & $\begin{array}{r}488.807 \\
1.254 .505\end{array}$ \\
\hline $\begin{array}{l}\text { Consulta } \\
\text { mental }\end{array}$ & $\begin{array}{l}\text { Realizó consulta mental } \\
\text { No realizó consulta mental }\end{array}$ & $\begin{array}{r}24.064 \\
831.617\end{array}$ & $\begin{array}{l}35 \% \\
50 \%\end{array}$ & $\begin{array}{r}44.347 \\
843.274\end{array}$ & $\begin{array}{l}65 \% \\
50 \%\end{array}$ & $\begin{array}{r}68.421 \\
1.674 .891\end{array}$ \\
\hline $\begin{array}{l}\text { Examen } \\
\text { laboratorio }\end{array}$ & $\begin{array}{l}\text { Sin exámenes } \\
\text { Con exámenes }\end{array}$ & $\begin{array}{l}550.887 \\
304.594\end{array}$ & $\begin{array}{l}53 \% \\
43 \%\end{array}$ & $\begin{array}{l}479.258 \\
408.573\end{array}$ & $\begin{array}{l}47 \% \\
57 \%\end{array}$ & $\begin{array}{r}1.030 .145 \\
713.167\end{array}$ \\
\hline $\begin{array}{l}\text { Quintil de } \\
\text { ingresos }\end{array}$ & $\begin{array}{l}\text { Quintil de ingreso } 1 \\
\text { Quintil de ingreso } 2 \\
\text { Quintil de ingreso } 3 \\
\text { Quintil de ingreso } 4 \\
\text { Quintil de ingreso } 5\end{array}$ & $\begin{array}{l}175.147 \\
179.918 \\
189.376 \\
170.842 \\
140.398\end{array}$ & $\begin{array}{l}42 \% \\
46 \% \\
50 \% \\
52 \% \\
61 \%\end{array}$ & $\begin{array}{c}244.396 \\
207.943 \\
187.195 \\
159.866 \\
88.231\end{array}$ & $\begin{array}{l}58 \% \\
54 \% \\
50 \% \\
48 \% \\
39 \%\end{array}$ & $\begin{array}{l}419.543 \\
387.861 \\
376.571 \\
330.708 \\
228.629\end{array}$ \\
\hline Región & $\begin{array}{l}\text { Región Metropolitana } \\
\text { Otra Región }\end{array}$ & $\begin{array}{l}321.009 \\
534.672\end{array}$ & $\begin{array}{l}48 \% \\
50 \%\end{array}$ & $\begin{array}{l}353.944 \\
533.687\end{array}$ & $\begin{array}{l}52 \% \\
50 \%\end{array}$ & $\begin{array}{r}674.953 \\
1.068 .359\end{array}$ \\
\hline
\end{tabular}

Fuente: Elaboración propia a partir de los datos de la Casen, 2015.

de educación formal (63\%), presentaban patologías asociadas a trastornos del ánimo (52\%), no presentan patología $(56 \%)$, reconocían no recibir ayuda de familiares o cercanos (74\%), presentaban sordera o dificultad para oír $(51 \%)$, ceguera o dificultad para ver (51\%), recibían alimentos del Programa de Alimentación Complementaria del Adulto Mayor (PACAM) (57\%), habían realizado consulta mental (65\%), estaban calificados en los quintiles de ingresos 1,2 y $3(58 \%, 54 \%, 50 \%)$, habitan mayoritariamente en la Región Metropolitana (52\%) (Tabla 2).

Los hombres y mujeres mayores que solicitan ayuda aumentaban en $94 \%$ y $100 \%$, respectivamente, la probabilidad de tener una mejor autopercepción de salud. También, hombres y mujeres mayores sin problemas de salud aumentaron en $64 \%$ y $48 \%$, respectivamente, la probabilidad de 
Tabla 3. Modelo de regresión logística estimado

\begin{tabular}{|c|c|c|c|c|c|c|c|}
\hline & \multirow{2}{*}{$\begin{array}{l}\text { Variables } \\
\text { Variables }\end{array}$} & \multicolumn{3}{|c|}{ Hombres } & \multicolumn{3}{|c|}{ Mujeres } \\
\hline & & $\begin{array}{l}\text { Odds } \\
\text { Ratio }\end{array}$ & $\begin{array}{l}\text { Signifi- } \\
\text { cación }\end{array}$ & $\begin{array}{l}\text { Intervalos de } \\
\text { Confianza Odds } \\
\text { (95\% Conf.) }\end{array}$ & $\begin{array}{l}\text { Odds } \\
\text { Ratio }\end{array}$ & $\begin{array}{l}\text { Signifi- } \\
\text { cación }\end{array}$ & $\begin{array}{l}\text { Intervalos de } \\
\text { Confianza Odds } \\
\text { (95\% Conf.) }\end{array}$ \\
\hline Nacionalidad & $\begin{array}{l}\text { Chileno } \\
\text { Extranjero }\end{array}$ & $\begin{array}{c}1,29 \\
---\end{array}$ & 0,000 & $\begin{array}{c}1,21-1,39 \\
---\end{array}$ & $\begin{array}{c}1,95 \\
---\end{array}$ & 0,000 & $1,85-2,06$ \\
\hline Educación & $\begin{array}{l}\text { Asistió a la educación formal } \\
\text { No asistió a la educación formal }\end{array}$ & 1,34 & 0,000 & $\begin{array}{c}1,31-1,3 \\
---\end{array}$ & $\begin{array}{c}1,27 \\
---\end{array}$ & 0,000 & $1,25-1,29$ \\
\hline Trabajo & $\begin{array}{l}\text { Con trabajo } \\
\text { Sin trabajo }\end{array}$ & 1,24 & 0,000 & $1,23-1,26$ & 1,21 & 0,000 & $1,19-1,22$ \\
\hline $\begin{array}{l}\text { Sistema } \\
\text { previsional }\end{array}$ & $\begin{array}{l}\text { Fonasa } \\
\text { Privado }\end{array}$ & 1,16 & 0,000 & $\begin{array}{c}1,14-1,18 \\
---\end{array}$ & 1,19 & 0,000 & $1,17-1,21$ \\
\hline Patología & $\begin{array}{l}\text { Cardiovascular } \\
\text { Trastornos del ánimo } \\
\text { Otra patología } \\
\text { Sin patología }\end{array}$ & $\begin{array}{l}0,85 \\
0,82 \\
0,62 \\
----\end{array}$ & $\begin{array}{l}0,000 \\
0,000 \\
0,000 \\
----\end{array}$ & $\begin{array}{c}0,84-0,86 \\
0,78-0,87 \\
0,61-0,63 \\
---\end{array}$ & $\begin{array}{c}0,72 \\
0,69 \\
0,55 \\
----\end{array}$ & $\begin{array}{l}0,000 \\
0,000 \\
0,000 \\
----\end{array}$ & $\begin{array}{l}0,71-0,73 \\
0,68-0,72 \\
0,54-0,56\end{array}$ \\
\hline $\begin{array}{l}\text { Redes de } \\
\text { apoyo }\end{array}$ & $\begin{array}{l}\text { Solicita ayuda } \\
\text { No solicita ayuda }\end{array}$ & 1,94 & 0,000 & $1,91-1,97$ & $\begin{array}{c}2,02 \\
---\end{array}$ & 0,000 & $1,99-2,04$ \\
\hline $\begin{array}{l}\text { Condición } \\
\text { permanente }\end{array}$ & $\begin{array}{l}\text { Dificultad física } \\
\text { Sordera o dificultad para oír } \\
\text { Ceguera o dificultad para ver } \\
\text { Otra condición permanente } \\
\text { Sin condición permanente }\end{array}$ & $\begin{array}{l}0,46 \\
0,79 \\
0,61 \\
0,56 \\
---\end{array}$ & $\begin{array}{l}0,000 \\
0,000 \\
0,000 \\
0,000 \\
---\end{array}$ & $\begin{array}{c}0,45-0,46 \\
0,77-0,81 \\
0,59-0,62 \\
0,53-0,58 \\
----\end{array}$ & $\begin{array}{l}0,45 \\
0,82 \\
0,72 \\
0,49 \\
---\end{array}$ & $\begin{array}{l}0,000 \\
0,000 \\
0,000 \\
0,000 \\
---\end{array}$ & $\begin{array}{c}0,43-0,46 \\
0,80-0,84 \\
0,70-0,73 \\
0,48-0,51 \\
----\end{array}$ \\
\hline $\begin{array}{l}\text { Problemas } \\
\text { de Salud }\end{array}$ & $\begin{array}{l}\text { Sin problemas de salud } \\
\text { Con problemas de salud }\end{array}$ & $\begin{array}{l}1,64 \\
----\end{array}$ & 0,000 & $1,62-1,66$ & $\begin{array}{c}1,48 \\
---\end{array}$ & 0,000 & $\begin{array}{c}1,46-1,49 \\
---\end{array}$ \\
\hline Nutrición & $\begin{array}{l}\text { Recibe alimentos del PACAM } \\
\text { No recibe alimentos del PACAM }\end{array}$ & $\begin{array}{c}1,16 \\
---\end{array}$ & $\begin{array}{c}0,000 \\
---\end{array}$ & $\begin{array}{c}1,14-1,17 \\
---\end{array}$ & $\begin{array}{c}1,04 \\
---\end{array}$ & $\begin{array}{c}0,000 \\
---\end{array}$ & $1,02-1,04$ \\
\hline $\begin{array}{l}\text { Consulta } \\
\text { mental }\end{array}$ & $\begin{array}{l}\text { Realizó consulta mental } \\
\text { No realizó consulta mental }\end{array}$ & $\begin{array}{l}1,47 \\
----\end{array}$ & 0,000 & $1,42-1,53$ & $\begin{array}{l}1,24 \\
----\end{array}$ & 0,000 & $1,21-1,27$ \\
\hline $\begin{array}{l}\text { Examen de } \\
\text { laboratorio }\end{array}$ & $\begin{array}{l}\text { Sin exámenes } \\
\text { Con exámenes }\end{array}$ & $\begin{array}{l}1,17 \\
----\end{array}$ & 0,000 & $\begin{array}{c}1,16-1,18 \\
---\end{array}$ & $\begin{array}{c}1,20 \\
---\end{array}$ & 0,000 & $\begin{array}{c}1,19-1,21 \\
---\end{array}$ \\
\hline $\begin{array}{l}\text { Quintil de } \\
\text { ingreso }\end{array}$ & $\begin{array}{l}\text { Quintil de ingreso } 1 \\
\text { Quintil de ingreso } 2 \\
\text { Quintil de ingreso } 3 \\
\text { Quintil de ingreso } 4 \\
\text { Quintil de ingreso } 5\end{array}$ & $\begin{array}{l}0,43 \\
0,57 \\
0,67 \\
0,70 \\
---\end{array}$ & $\begin{array}{l}0,000 \\
0,000 \\
0,000 \\
0,000 \\
---\end{array}$ & $\begin{array}{c}0,42-0,44 \\
0,56-0,59 \\
0,66-0,68 \\
0,69-0,72 \\
---\end{array}$ & $\begin{array}{l}0,61 \\
0,65 \\
0,72 \\
0,69 \\
---\end{array}$ & $\begin{array}{l}0,000 \\
0,000 \\
0,000 \\
0,000 \\
---\end{array}$ & $\begin{array}{c}0,6-0,62 \\
0,64-0,67 \\
0,71-0,73 \\
0,67-0,70 \\
---\end{array}$ \\
\hline Región & $\begin{array}{l}\text { Tarapacá } \\
\text { Antofagasta } \\
\text { Atacama } \\
\text { Coquimbo } \\
\text { Valparaíso } \\
\text { O'Higgins } \\
\text { Maule } \\
\text { Biobío } \\
\text { Araucanía } \\
\text { Los Lagos } \\
\text { Aysen } \\
\text { Magallanes } \\
\text { Metropolitana } \\
\text { Los Ríos } \\
\text { Arica y Parinacota }\end{array}$ & $\begin{array}{l}1,41 \\
0,96 \\
0,91 \\
1,13 \\
1,42 \\
1,06 \\
1,03 \\
1,45 \\
1,29 \\
1,46 \\
1,66 \\
0,89 \\
--- \\
1,41 \\
1,22\end{array}$ & $\begin{array}{l}0,000 \\
0,040 \\
0,000 \\
0,000 \\
0,000 \\
0,000 \\
0,009 \\
0,000 \\
0,000 \\
0,000 \\
0,000 \\
0,000 \\
---- \\
0,000 \\
0,000\end{array}$ & $\begin{array}{r}1,34-1,48 \\
0,93-0,99 \\
0,87-0,96 \\
1,10-1,16 \\
1,39-1,44 \\
1,04-1,09 \\
1,01-1,04 \\
1,43-1,47 \\
1,27-1,32 \\
1,42-1,49 \\
1,54-1,79 \\
0,84-0,94 \\
--- \\
1,36-1,45 \\
1,16-1,28\end{array}$ & $\begin{array}{l}1,82 \\
1,77 \\
1,19 \\
1,13 \\
1,39 \\
0,96 \\
1,13 \\
1,31 \\
1,20 \\
1,58 \\
1,55 \\
1,68 \\
--- \\
1,36 \\
1,14\end{array}$ & $\begin{array}{l}0,000 \\
0,000 \\
0,000 \\
0,000 \\
0,000 \\
0,067 \\
0,000 \\
0,000 \\
0,000 \\
0,000 \\
0,000 \\
0,000 \\
--- \\
0,000 \\
0,000\end{array}$ & $\begin{array}{r}1,74-1,90 \\
1,72-1,83 \\
1,15-1,24 \\
1,11-1,16 \\
1,38-1,42 \\
0,95-0,98 \\
1,11-1,15 \\
1,29-1,32 \\
1,18-1,22 \\
1,55-1,61 \\
1,45-1,66 \\
1,59-1,75 \\
\quad--- \\
1,32-1,40 \\
1,09-1,20\end{array}$ \\
\hline Constante & & 0,10 & 0,000 & $0,09-0,11$ & 0,13 & 0,000 & $0,12-0,14$ \\
\hline
\end{tabular}

Fuente: Elaboración propia a partir de los datos de la Casen, 2015. 
tener una mejor autopercepción de salud. Red de apoyo y problemas de salud fueron las dos variables que presentaron la mayor magnitud estadística del estudio (Tabla 3 ).

Tener la nacionalidad chilena incrementó la razón de buen estado de salud autorreportado en 1,29 y 1,95 veces, para hombres y mujeres, con respecto a ser un hombre o mujer extranjero mayor (Tabla 3).

El haber asistido a la educación formal aumentó la razón de buen estado de salud autorreportado en 1,34 y 1,27 veces, para hombres y mujeres, con respecto al mayor que sí participó del sistema de educación formal (Tabla 3 ).

El haber trabajado como mínimo una hora en la semana anterior a la entrevista, incrementó la razón de buen estado de salud autorreportado en 1,24 y 1,21 veces, para hombres y mujeres, con respecto al mayor que no había trabajado (Tabla 3).

Participar del sistema previsional Fonasa incrementó la razón de buen estado de salud autorreportado en 1,16 y 1,19 veces, para hombres y mujeres, con respecto al mayor que estaba afiliado del sistema de financiamiento de salud privada. Tabla 3.

Un mayor que recibe ayuda de un familiar incrementó la razón de buen estado de salud autorreportado en 1,94 y 2,02 veces, para los hombres y mujeres, con respecto a los que no recibieron ayuda (Tabla 3 ).

Un adulto mayor que no tuvo problemas de salud incrementó la razón buen estado de salud autorreportado en 1,64 y 1,48 veces, para hombres y mujeres, con respecto a quien declaró poseer problemas de salud (Tabla 3 ).

Recibir alimentos a través del sistema de salud público incrementó la razón de buen estado de salud autorreportado en 1,16 y 1,04 veces, para hombres y mujeres, con respecto al mayor que no los recibió (Tabla 3 ).

Haber realizado una consulta mental incrementó la razón de buen estado de salud autorreportado en 1,47 y 1,24 veces, para hombres y mujeres, con respecto al mayor que no la hubiera realizado (Tabla 3 ).

El hecho de no haberse realizado exámenes de laboratorio incrementó la razón de buen estado de salud autorreportado en 1,17 y 1,20 veces para hombres y mujeres, con respecto al mayor que sí los había realizado (Tabla 3).
Padecer una patología cardiovascular, trastornos del ánimo u otro problema de salud disminuyó la razón de buen estado de salud autorreportado en 0,$85 ; 0,82 ; 0,62$ y $072 ; 0.69 ; 0,55$ veces, respectivamente, para hombres y mujeres, respectivamente, en comparación con el mayor que no presentaba problemas de salud (Tabla 3).

Padecer la condición permanente de dificultad física, sordera, ceguera u otra condición disminuyó la razón de buen estado de salud autorreportado en 0,$46 ; 0,79 ; 0,61 ; 0,56$ y 0,$45 ; 0,82 ; 0,72$ y 0,49 veces, respectivamente, para hombres y mujeres, con respecto al mayor que no presentaba una condición permanente de dificultades físicas (Tabla 3).

Pertenecer a los quintiles de ingreso 1, 2, 3 y 4 disminuía la razón de buen estado de salud autorreportado en 0,$43 ; 0,57 ; 0,67$ y 0,7 y 0,$61 ; 0,65$; 0,72 y 0,69 veces, respectivamente, para hombres y mujeres, con respecto a quienes pertenecían al último quintil de ingresos (Tabla 3).

Para los hombres, habitar en las regiones de Aysén, Los Lagos, Biobío o Valparaíso incrementaba la razón de buen estado de salud autorreportado en 1,$66 ; 1,46 ; 1.45$ y 1,42 veces. Sin embargo, para las mujeres, habitar en las regiones de Tarapacá, Antofagasta, Magallanes o Los Lagos incrementaba la razón de buen estado de salud autorreportado en 1,$82 ; 1,77 ; 1,68 ; 1,58$, veces, respectivamente. Lo anterior, con respecto a los mayores que habitaban en la Región Metropolitana (Tabla 3).

\section{Discusión}

En Chile existe un estudio que emplea la Casen 2015 para efectos de investigar la equidad en el acceso a salud en Chile ${ }^{14}$. Paralelamente, la Encuesta Nacional de Calidad de Vida en la Vejez (2010) mide la autopercepción de salud en la dimensión "condiciones de salud"15, mostrando que la percepción de los mayores sobre el buen estado de salud mejoró al año 2010, con respecto al año 2007.

También, existen estudios que muestran que analizar el estado de salud de los mayores como grupo homogéneo es una limitante, porque puede ocultar la naturaleza de las necesidades, las diferencias sociales y de género en salud ${ }^{16}$. Dichos estudios muestran que la percepción de mala sa- 
lud fue superior en las mujeres, en línea con los resultados del presente estudio. En América Latina existen estudios que muestran que la autopercepción de la salud de los mayores está asociada positivamente con mayor educación ${ }^{17}$. También, el presente estudio para mujeres y hombres mayores el haber asistido a la educación formal incrementa la razón de buen estado de salud.

Asimismo, se ha planteado que la autopercepción positiva de salud se explica, en parte, por el tipo de enfermedad que padece el mayor y el apoyo de su entorno próximo ${ }^{18}$. Este estudio muestra que las personas con patologías cardiovasculares o asociadas al trastorno del ánimo reportan un mal estado de salud, y quienes cuentan con redes de apoyo tienen un estado de salud autorreportado positivo.

También existen estudios que muestran que una buena nutrición y un estilo de vida activo influyen positivamente en la autopercepción de salud de los mayores ${ }^{19-20}$. Este estudio muestra que los mayores que reciben alimentos del Programa PACAM presentan un estado de salud autorreportado más favorable que aquellos mayores que no lo hacen, lo anterior, logra uno de los objetivos del programa, que es contribuir a mantener el estado nutricional y la funcionalidad de los mayores y optimizar su calidad de vida ${ }^{21}$.

Se muestra que un mayor que realiza consulta mental presenta un mejor estado de salud autorreportado, en relación a un mayor que no lo hace. El deterioro cognitivo y demencias alzan su prevalencia en la medida que aumenta la edad. Se puede inferir, pero no asegurar, que la consulta mental en los mayores actúa como una medida preventiva. Es importante señalar que la mayor prevalencia de demencia en Chile se ubica en niveles socioculturales bajos y en población rural ${ }^{22}$.

Existen estudios que muestran que el nivel socioeconómico y la autopercepción de la salud de los mayores están estrechamente asociados entre $s i^{23-25}$. Este trabajo muestra que los mayores que pertenecen a los quintiles de ingreso más bajos tienen más baja autopercepción de salud, con respecto al quinto quintil de ingresos más alto. Se puede inferir que los mayores presentan una relación estrecha entre estado de salud autorreportado y quintil de ingreso.

Finalmente, teniendo presente el diseño de un modelo de decisión para evaluar el impacto de la políticas públicas, se puede inferir que los factores sociales que explican el estado de salud autorreportado en los mayores se pueden agrupar multidimensionalmente en cuatro dimensiones: cultural, económica, salud y entorno. En la dimensión cultural, los factores relevantes son: nacionalidad, educación y región en que se habita. En la dimensión económica son: trabajo y quintil de ingresos. En la dimensión salud: sistema de atención sanitaria pública, las patologías cardiovasculares y trastornos del ánimo, condición de patología permanente, nutrición, salud mental y control preventivo usando exámenes de laboratorio. En la dimensión entorno se encuentran las redes de apoyo.

También se puede inferir que el buen estado de salud autorreportado por los mayores en Chile es preferentemente masculino, en una sociedad que presenta un envejecimiento muy heterogéneo y mayoritariamente femenino.

\section{Referencias}

1. Villalobos P. Panorama de la dependencia en Chile: avances y desafíos. Rev Med Chile 2019; 147: 83-90.

2. Saldivia S, Vicente B, Torres F. Medición de resultados en evaluaciones económicas. Rev Med Chile 2010; 138 (Supl 2): 79-82.

3. Meléndez M, Montero R, Jiménez C, Blanco L. Autopercepción de salud en ancianos no institucionalizados. Atención Primaria 2001; 28 (2): 91-6.

4. Savassi L. A satisfação do usuário e a autopercepção da saúde em atenção primária. Rev Bras Med Fam Comunidade 2010; 5 (17): 3-5.

5. Dutra F, Costa L, Sampaio R. A influência do afastamento do trabalho na percepção de saúde e qualidade de vida de indivíduos adultos. Fisioter Pesqui 2016; 23 (1): 98-104.

6. Gallegos K, García C, Durán C, Reyes H, Durán L. Autopercepción del estado de salud del estado de salud: un aproximación a los ancianos de México. Rev Saúde Pública 2006; 40 (5): 792-801.

7. Riveros C, Rodríguez P, Palomo R, Alvear S, Fernández MA, Arenas A. El maltrato estructural a personas mayores en Chile y la necesidad de formular un índice multidimensional. UNIVERSUM 2017; 32 (2): 163-76.

8. Encuesta de Caracterización Socioeconómica Nacional (CASEN). 2015. Acceso: 14/11/2017. Disponible en:http://observatorio.ministeriodesarrollosocial.gob.cl/ casen-multidimensional/casen/casen_2015.php

9. Segovia J, Bartiett R, Edwards A. An empirical analysis 
of the dimensions of healt status measures. Soc Sci Med 1989; 29: 761-8.

10. Mossey J, Shapiro E. Self -rated: a predictor of mortality among the elderly. Public Health 1982; 72: 800-8.

11. Peña C. (2012). "Ëtica y derecho en la investigación en ciencias sociales" en Bioética en Investigación en Ciencias Sociales. Acceso: 14/11/2017. Disponible en: http:// www.conicyt.cl/fondecyt/files/2012/10/Libro-3-Bio\%C3\%A9tica-en-investigaci\%C3\%B3n-en-ciencias-sociales.pdf.

12. Ley No 19.828; Convención Interamericana sobre Protección de Derechos de Personas Mayores, 2011. Acceso: 14/11/217. Disponible en: Biblioteca del Congreso Nacional. https://www.leychile.cl/Navegar?idNor$\mathrm{ma}=202950$.

13. Collett D. Modelling Binary Data. Chapman and Hall. London, 1991.

14. Frenz P, Delgado I, Villanueva J, Muñoz F, Navarrete M, Kaufman J. Salud y Equidad. Análisis de la Equidad en el acceso a la salud en Chile con datos de la encuesta CASEN. Rev Med Chile 2013; 141 (9): 1095-106.

15. Encuesta Calidad de Vida en la vejez, UC-Caja de compensación Los Andes, (2017). Acceso: 14/11/2017. Disponible en: https://www.cooperativa.cl/noticias/site/ artic/20170429/asocfile/20170429174326/iv_encuesta_de_calidad_de_vida_en_la_vejez.pdf.

16. Séculi E, Fusté J, Brugulat P, Juncá Rué M, Guillén M. Percepción del estado de salud en varones y mujeres en las últimas etapas de la vida. Gac Sanit 2001; 15 (3): 217-23.

17. Jewell T, Rossi M, Triunfo P. El estado de Salud del Persona Mayor en América Latina. Cuad Econ 2007; 26
(46): 147-67.

18. Peláez E, Acosta D, Laura C. Factores asociados a la autopercepción de salud en adultos mayores. Revista Cubana de Salud Pública 2015; 41(4): 638-48.

19. Organización Mundial de la Salud (OMS). 2015. Informe Mundial sobre el envejecimiento y la salud. Acceso: 14/11/2017. Disponible en: http://www.afro.who.int/ sites/default/files/2017-06/9789240694873_spa.pdf.

20. Osorio P, Torrejón M, Anigstein M. Calidad de vida en personas mayores en Chile. Revista Mad-Universidad de Chile 2011; 24: 61-75.

21. Ceroni P, Alvear S, Pino G. Determinantes de no-participación en el programa de alimentación complementaria de personas mayores, resultados de la CASEN 2015. Rev Chil Nutr 2019; 46 (1): 50-6.

22. Servicio Nacional del Persona Mayor-SENAMA (2010): Estudio Nacional de la Dependencia en las personas mayores. Acceso: 14/11/2017. Disponible en:http://www. superacionpobreza.cl/wp-content/uploads/2014/01/ estudiodependencia.pdf.

23. Subramanian V, Delgado I, Jadue L, Kawachi I, Vega J. Inequidad de ingreso y autopercepción de salud: un análisis desde la perspectiva contextual en las comunas chilenas. Rev Med Chile 2003; 131: 321-30.

24. Venkata S, Delgado I, Jadue L, Kawachi I, Vega J. Inequidad de ingreso y autopercepción de salud: un análisis desde la perspectiva contextual en las comunas chilenas. Revista Med Chile 2003; 131(3): 321-30.

25. Todd J, Rossi M, Triunfo P. El estado de salud del Persona Mayor en américa latina. Cuadernos de Economía 2007; 26(46): 147-67. 